\title{
Hypopigmentation-punctate palmoplantar keratoderma syndrome
}

INSERM

\section{Source}

INSERM. (1999). Orphanet: an online rare disease and orphan drug data base.

Hypopigmentation-punctate palmoplantar keratoderma syndrome. ORPHA:324561

A rare, genetic, epidermal disease characterized by punctate keratoderma on palms and soles associated with irregularly shaped hypopigmented macules (typically localized on the extremities). Ectopic calcification (e.g. early-onset calcific tendinopathy, calcinosis cutis) and pachyonychia may be occasionally associated. 